\title{
Crosstalk between osteoblasts and endothelial cells co-cultured on a polycapro- lactone-starch scaffold and the in vitro development of vascularization
}

\author{
Marina I. Santos ${ }^{\text {a,b,c }}$, Ronald E. Unger ${ }^{c}$, Rui A. Sousa ${ }^{\text {a,b }}$, Rui L. Reis ${ }^{\text {a,b }}$, C. James Kirkpatrick ${ }^{\text {c,* }}$ \\ a 3B's Research Group - Biomaterials, Biodegradables and Biomimetics, University of Minho, Headquarters of the European Institute of Excellence \\ on Tissue Engineering and Regenerative Medicine, AvePark, 4806-909 Taipas, Guimarães, Portugal \\ ${ }^{\mathrm{b}}$ IBB - Institute for Biotechnology and Bioengineering, PT Associated Laboratory, Guimarães, Portugal \\ ${ }^{\mathrm{c}}$ Institute of Pathology, Johannes Gutenberg University Mainz, Langenbeckstr. 1, Mainz 55101, Germany
}

\section{A R T I C L E I N F O}

\section{Article history:}

Received 4 March 2009

Accepted 5 May 2009

Available online 31 May 2009

\section{Keywords:}

Co-culture

Vascularization

Bone

Tissue engineering

Polymer

\begin{abstract}
A B S T R A C T
The reconstruction of bone defects based on cell-seeded constructs requires a functional microvasculature that meets the metabolic demands of the engineered tissue. Therefore, strategies that augment neovascularization need to be identified. We propose an in vitro strategy consisting of the simultaneous culture of osteoblasts and endothelial cells on a starch-based scaffold for the formation of pre-vascular structures, with the final aim of accelerating the establishment of a vascular bed in the implanted construct. Human dermal microvascular endothelial cells (HDMECs) were co-cultured with human osteoblasts (hOBs) on a 3D starch-based scaffold and after 21 days of culture HDMEC aligned and organized into microcapillary-like structures. These vascular-like structures evolved from a cord-like configuration to a more complex branched morphology, had a lumen and stained in the perivascular region for type IV collagen. Genetic profiling of 84 osteogenesis-related genes was performed on coculture vs. monoculture. Osteoblasts in co-culture showed a significant up-regulation of type I collagen and immunohistochemistry revealed that the scaffold was filled with a dense matrix stained for type I collagen. In direct contact with HDMEC hOBs secreted higher amounts of VEGF in relation to monoculture and the highest peak in the release profile correlated with the formation of microcapillary-like structures. The heterotypic communication between the two cell types was also assured by direct cellcell contact as shown by the expression of the gap junction connexin 43. In summary, by making use of heterotypic cellular crosstalk this co-culture system is a strategy to form vascular-like structures in vitro on a 3D scaffold.
\end{abstract}

(c) 2009 Elsevier Ltd. All rights reserved.

\section{Introduction}

The reconstruction of large skeletal defects, such as those resulting from resection of bone tumors or trauma, is still a major orthopedic challenge. Tissue engineering strategies often fail to regenerate these defects due to inadequate vascularization [1]. A functional microvasculature supplying the construct would guarantee the metabolic demand of the seeded cells, of the newly formed tissue, and could participate in other as yet unknown aspects of cellular crosstalk in orchestrating bone formation. Due to their unique role in angiogenesis, endothelial cells (ECs) have attracted most attention in strategies that seek to achieve bone vascularization. However, there is clear evidence that on their own these cells cannot do more than form incipient vascular structures

\footnotetext{
* Corresponding author. Tel.: +49 6131 177301; fax: +49 613117477301.

E-mail address: kirkpatrick@ukmainz.de (C.J. Kirkpatrick).
}

that resemble early capillaries but which are not stable in the longterm $[2,3]$. Therefore, ECs co-cultured with the cell type characteristic of the tissue to be regenerated has been a proposed strategy. Hence, since bone is a tissue formed by several cell types, cocultures of heterogeneous cells recreates more closely the in vivo microenvironment than monotypic cell cultures. Several studies have shown that there is a reciprocal regulation and functional relationship between ECs and osteoblasts during osteogenesis [4]. Numerous regulatory molecules (e.g. endothelins, prostaglandins) which exert major effects in controlling the differentiation and activity of bone-forming cells are secreted by ECs [5]. On the other hand, osteoblasts influence EC activity through the release of diverse angiogenic growth factors, such as VEGF and bFGF [6]. Furthermore, cell-cell interaction mediated by proteins at gap junctions is another communication strategy used between these two cell types [7].

Previously, our group [8] showed that co-culturing ECs derived from the microvasculature (human dermal microvascular 
endothelial cells, HDMECs) with primary human osteoblasts (hOBs) resulted in the formation of microcapillary-like structures similar to those observed in vivo. Surprisingly, these two cell populations were able to "self-assemble" from a cell suspension mixture seeded on the three-dimensional biomaterials investigated. Other models of coculture for bone tissue engineering have been also proposed [9-12], nevertheless most of the co-culture systems proposed to date are mainly models to study heterotypic interactions and only few include the use of 3D biomaterial scaffolds $[8,10]$. Hence, the goal of this study was to use a human co-culture system directly on established 3D scaffolds to investigate more closely the crosstalk between osteoblasts and vascular cells in forming microvessels within the three-dimensionality of an innovative biomaterial. HDMECs:hOBs were co-cultured on fiber-mesh scaffolds made from a blend of starch with polycaprolactone (SPCL), a 3D support previously proposed and extensively studied for bone tissue engineering [1320]. The co-culture system on polycaprolactone-starch scaffolds was evaluated as a strategy to unravel the mechanisms of formation of vascular-like structures in the context of biomaterials for bone regeneration. Hence, in the scope of this work specific questions related to cellular interactions were addressed: (i) the 3D cellular distribution and the dynamics of the two cell populations in the scaffolding material; (ii) gene expression profiling and (iii) heterotypic communication in co-culture. In addition, we do not rule out the possibility of a pre-seeding co-culture strategy, followed by implantation, but this was not the prime aim of our study, as the problem of rapidly connecting an in vitro formed microcirculatory network to the functional microcirculation in vivo has not yet been satisfactorily solved.

\section{Materials and methods}

\subsection{Scaffolds}

A blend of corn starch with poly( $\varepsilon$-caprolactone) (SPCL, 30/70 wt\%) was used to produce fiber-mesh scaffolds. SPCL fibers were produced by melt-spinning (mean fiber diameter of $213 \pm 50 \mu \mathrm{m}$ ), subsequently cut into $10 \mathrm{~mm}$ segments, subjected to thermal treatment and compressed and bonded into a porous mesh. Standardized scaffolds had a circular die $(0.6 \mathrm{~cm}$ in diameter) and exhibited a porosity of $67.9 \pm 1.6 \%$, as determined by micro-computed tomography. All samples were sterilized by ethylene oxide. More details about these scaffolds and their properties are published elsewhere $[15,16,20]$.

\subsection{Cells and culture conditions}

HDMECs were derived from juvenile foreskin and hOBs were isolated from human femoral head explants. The use of this biological material for research purposes was authorized by the responsible ethical committee and was based on informed consent. HDMECs were obtained by enzymatic digestion [21], and were cultured in supplemented Endothelial Basal Medium MV (PromoCell) whose composition is detailed in Ref. [13]. hOBs were obtained by sequential enzymatic digestion of bone chips [22] and cultured in supplemented DMEM medium with $1000 \mathrm{mg} / \mathrm{L}$ D-glucose [8].

\subsection{HDMECs:hOBs co-culture on SPCL fiber-mesh scaffolds}

HDMECs and hOBs were mixed in a proportion of 4:1 and cultured in HDMEC medium. Before cell seeding SPCL fiber-mesh scaffolds were pre-coated with fibronectin $(10 \mu \mathrm{g} / \mathrm{mL}$ PBS $)$ for $1 \mathrm{~h}$ at $37^{\circ} \mathrm{C}$. Subsequently, $1.5 \times 10^{5}$ cells of the mixed cell suspension were added to each scaffold and cultured for up to 35 days. Scaffolds were also cultured with HDMECs $\left(1.30 \times 10^{5}\right.$ cells/scaffold $)$ or hOBs $\left(2 \times 10^{4}\right.$ cells/ scaffold) alone to be used as monoculture controls.

\subsection{Immunostaining of PECAM-1 (CD31) and CX43}

Samples were stained for PECAM-1 (CD31, endothelial-specific) and for nuclei (identifying both hOBs and ECs). Every 7 days of culture a co-culture sample and one sample from each monoculture control (HDMECs and hOBs) were fixed in a solution of $2 \%$ paraformaldehyde. Fixed and permeabilized samples ( $0.1 \%$ Triton) were incubated with the primary antibody mouse anti-human PECAM-1 (1:50, Dako) and then with the secondary antibody anti-mouse Alexa Fluor 488 (Invitrogen). For Cx43 a double staining was performed and the samples were incubated with PECAM-1 antibody and with rabbit anti-human Cx43 (1:50, Cell Signalling), followed by the incubation with goat anti-mouse Alexa Fluor 594 (for PECAM-1, red fluorescence) and anti-rabbit Alexa Fluor 488 (for Cx43, green fluorescence). The nuclei were counterstained with Hoechst, the samples mounted with Gel/Mount and visualized by CLSM (Leica TCSN NT).

\subsection{Real-time $P C R$}

After 21 days of culture, total RNA was extracted from co-cultures and monocultures on SPCL fiber-mesh scaffolds using the RNeasy Micro Kit (Qiagen). Total RNA $(0.5 \mu \mathrm{g})$ was reverse transcribed using Omniscript RT Kit (Qiagen). Equal amounts of cDNA (1.25 ng) plus master mix RT ${ }^{2}$ SYBR Green/ROX qPCR $(12.5 \mu \mathrm{L})$ were added in a final volume of $25 \mu \mathrm{L}$ to each well of the human osteogenesis $\mathrm{RT}^{2}$ Profiler PCR array for quantitative polymerase chain reaction (PCR) (Superarray). The human osteogenesis $\mathrm{RT}^{2}$ Profiler PCR array profiles the expression of 84 genes related to osteogenesis besides housekeeping and control genes. Gene amplification was performed using Applied Biosystems 7300 Real-Time PCR System. The number of cycles and annealing temperature were selected according to the manufacturer's instructions. For these experiments three different donors for both OBs and ECs were used. In each sample the mRNA level expression of each gene was normalized to the average expression of the housekeeping genes GAPDH and RPL13A. Gene fold change was calculated in comparison with HDMEC or hOBs as control samples. For each individual gene, fold differences in co-culture/monoculture were plotted in $\log _{2}$ scale against the associated statistic probability ( $p$ value in $\log _{10}$ scale). Two tailed Student's $t$-test was employed to detect significant differences in gene expression between co-culture and monoculture (OBs and ECs) experiments. Differences were considered statistically significant for $p<0.05$.

To verify the Superarray data obtained for Col I gene real-time PCR was performed with primers synthesized commercially: (forward) 5'-CTGGCCTCGGAGGAAACTTT-3' and (reverse) 5'-CCTCCGGTTGATTTCTCATCA-3'. RNA was isolated from four different HDMEC and hOB donors (three donors were the same as used for array analysis) and reverse transcribed into cDNA as previously described. Real-time PCR was performed with $2.5 \mathrm{ng}$ cDNA and $12.5 \mu \mathrm{L}$ of $2 \times$ master mix, primers $(0.25 \mu \mathrm{L}$ forward and $0.25 \mu \mathrm{L}$ reverse primer) in a final volume of $25 \mu \mathrm{L}$. Gene expression was normalized to the expression of the housekeeping gene GAPDH and gene fold change was calculated as previously explained.

\subsection{Immunohistochemical analysis}

HDMEC-hOB co-cultures on the biomaterial scaffold were prepared for immunohistochemistry as previously described [8]. Briefly, deparaffinized transversal cross-sections ( $5 \mu \mathrm{m}$ thick) were first incubated with mouse anti-human PECAM-1 rabbit anti-human Col I (1:100, Biodesign International) or mouse anti-human Col $\operatorname{IV}(1: 50$, Dako), and then incubated with the biotin-labeled secondary antibody horse anti-mouse or goat anti-rabbit. Detection of bound antibody was carried out by alkaline phosphatase staining and counterstained with Mayer's haematoxylin.

\subsection{Vascular endothelial growth factor (VEGF) quantification}

The supernatant of co-culture and monocultures on SPCL fiber-mesh scaffolds was collected and stored at $-80^{\circ} \mathrm{C}$ every 7 days. For each time point, three samples were taken and vascular endothelial growth factor (VEGF) was quantified by Enzyme-Linked Immunosorbent Assay (ELISA) using the human VEGF DuoSet (R\&D Systems) according to the manufacturer's protocol. The culture medium was used as blank.

\section{Results and discussion}

In the co-culture system under evaluation, which started from a mixed cell suspension of hOBs and EC seeded onto the biomaterial, the distribution of the two cell types in the scaffold reflected a heterotypic interaction, which was variable with time and involved a cellular self-assembly phenomenon especially prominent in the case of the HDMEC. While early time points (7 days) where characterized by cellular segregation of HDMECs appearing as monolayer patches above the osteoblasts (Fig. 1A), by 21 days of culture HDMECs had aligned and organized into microcapillary-like structures (Fig. 1B). In these structures, cells established contact through the cell-cell adhesion molecule PECAM-1, a homotypic contact known to be crucial for vessel formation and maintenance [23]. These vascular-like structures were established among osteoblasts, which deposited a dense matrix in such a manner that the microvessel-like cords were interwoven through the individual fibers of the scaffold. At day 35, microcapillary-like structures 

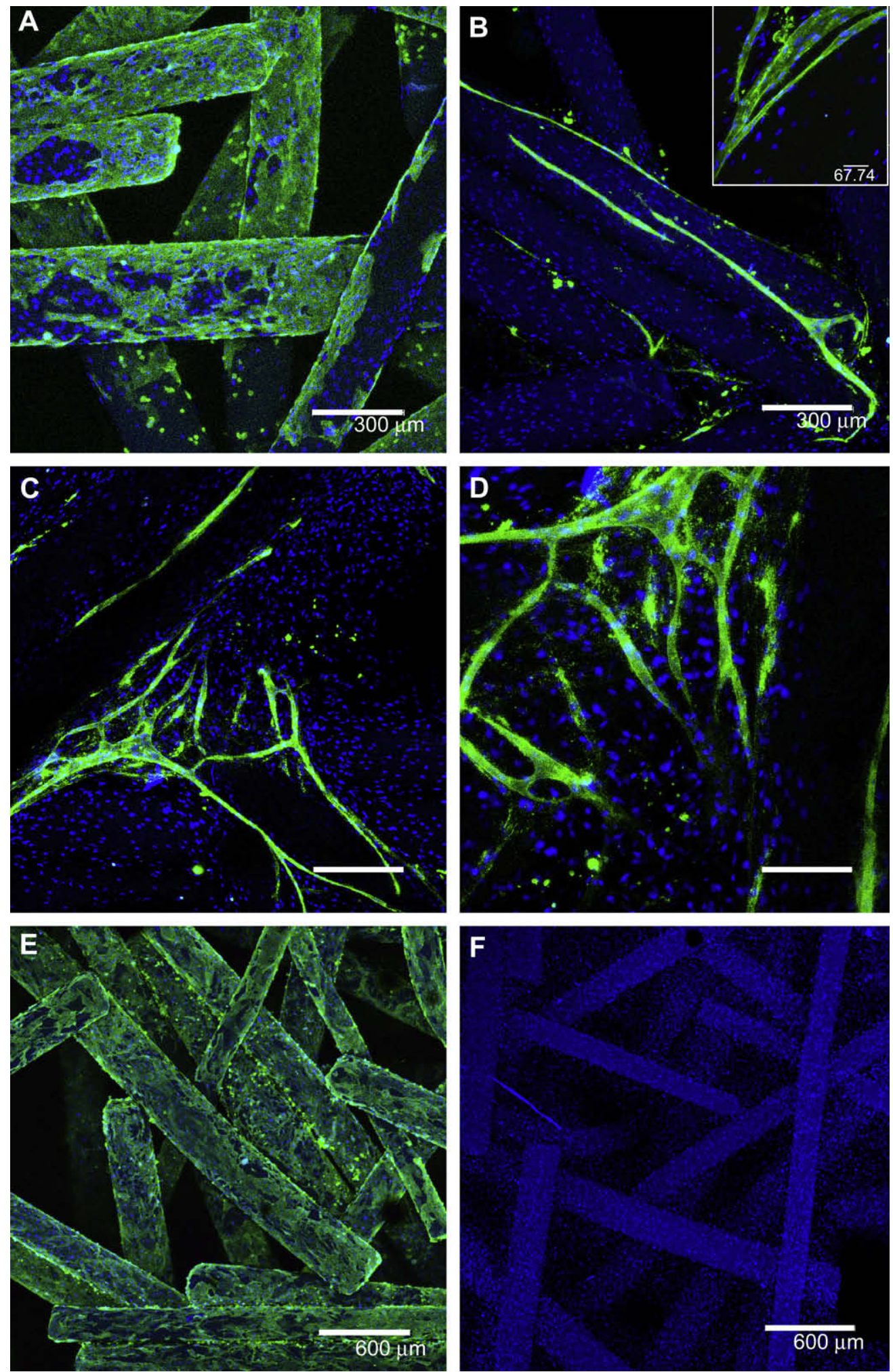

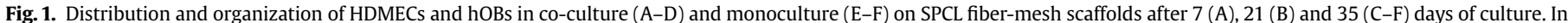

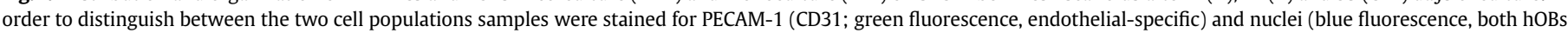

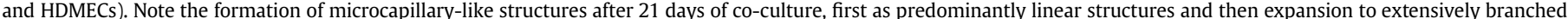

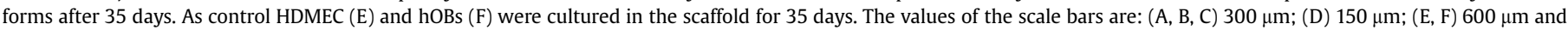
$67 \mu \mathrm{m}$ for the picture inserted in (B). 
exhibited several branching points, denoting a higher level of complexity (Fig. 1C-D). In monocultures, HDMEC cultured alone grew as a monolayer and no vascular-like structures were observed, while hOBs' monoculture grew extensively over the 35 days of culture, colonizing the entire scaffold surface and depositing matrix (Fig. 1E-F).

Immunohistochemistry performed on thin sections of SPCL fiber-mesh scaffolds after 35 days of co-culture unveiled other important aspects of these vascular-like structures, such as lumen formation, type IV collagen (Col IV) deposition and a dense surrounding type I collagen (Col I) matrix. Cross-sections in both the outer and inner parts of the scaffold revealed a dense network matrix which stained for Col I and occupied all the void spaces between fibers (Fig. 2A). A more intense Col I-staining was detected in the immediate surroundings of the microcapillary-like structures, which demonstrated a definitive lumen containing degenerating cells (Fig. 2B). Sections were also stained for markers of the endothelial phenotype such as PECAM-1 and Col IV. Cells stained positively for the endothelial marker PECAM-1 were organized in microcapillary-like structures forming a lumen (Fig. 2C, D). Lumen formation results from a complex molecular mechanism involving the up-regulation of over 1000 genes [24,25]. Furthermore, the areas corresponding to microcapillary-like structures were stained for Col IV, one of the hallmarks of the endothelial basement membrane (Fig. 2E, F). The overall morphological impression given in Fig. 2 is that of a tissue-like self-assembly of the EC in a matrix in which the osteoblasts are embedded, reminiscent of a histological micrograph (Fig. 2C). Col IV provides the major structural support
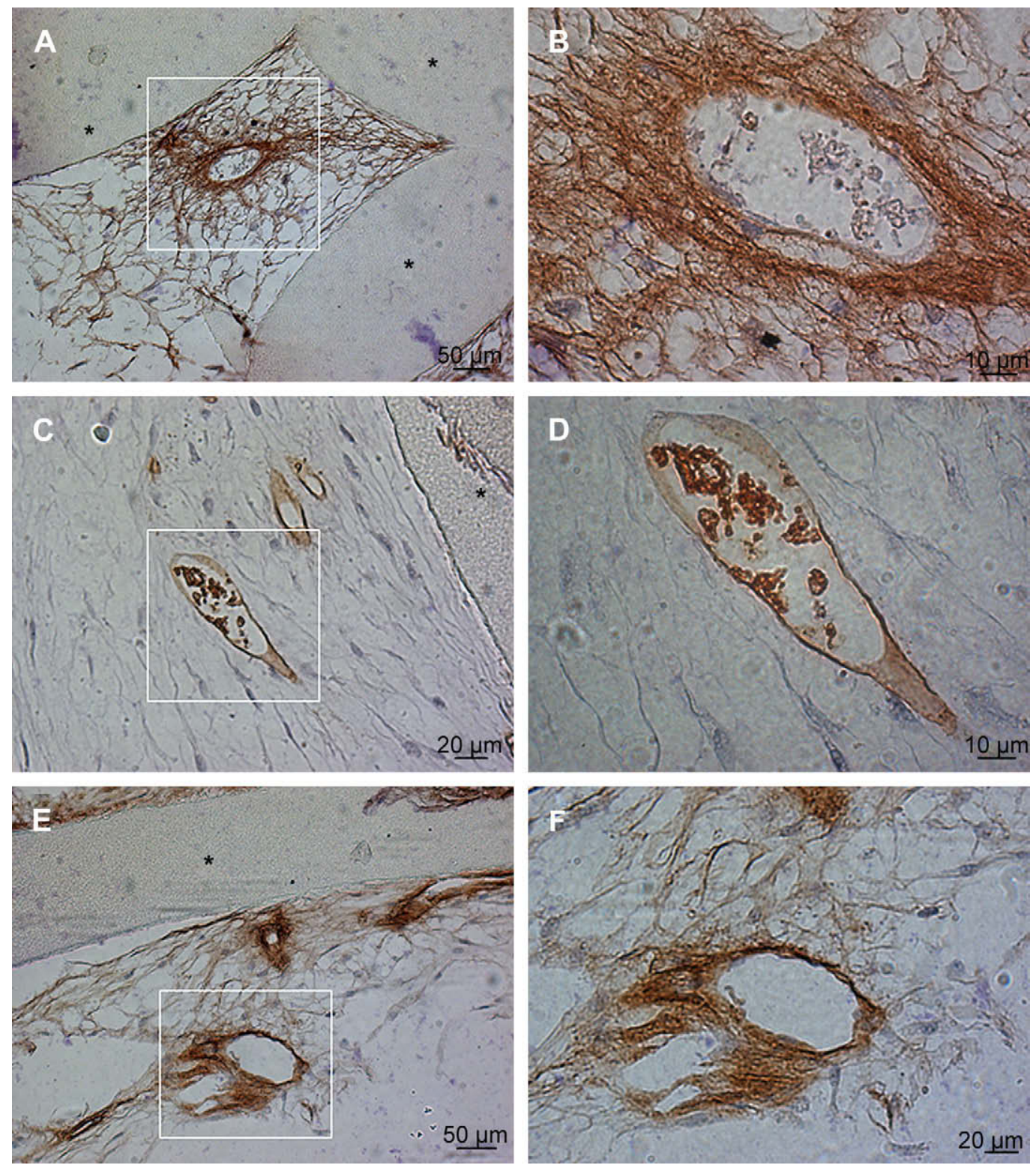

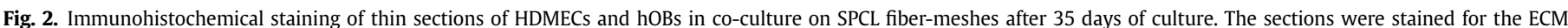

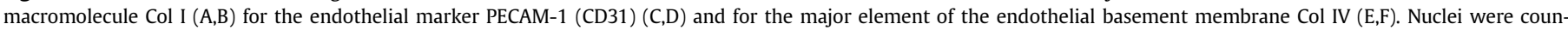

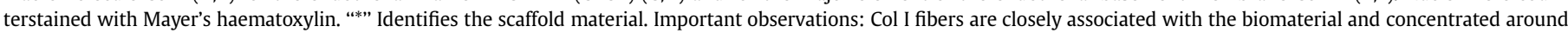

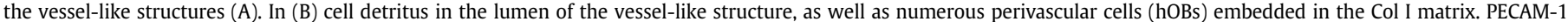

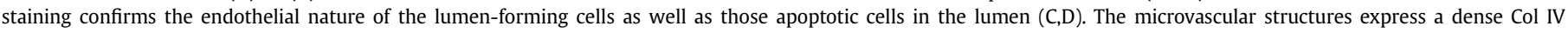
perivascular matrix (E,F). The values of the scale bars are: (A,E) $50 \mu \mathrm{m}$ and (B,C,D,F) $20 \mu \mathrm{m}$. 


\section{A}

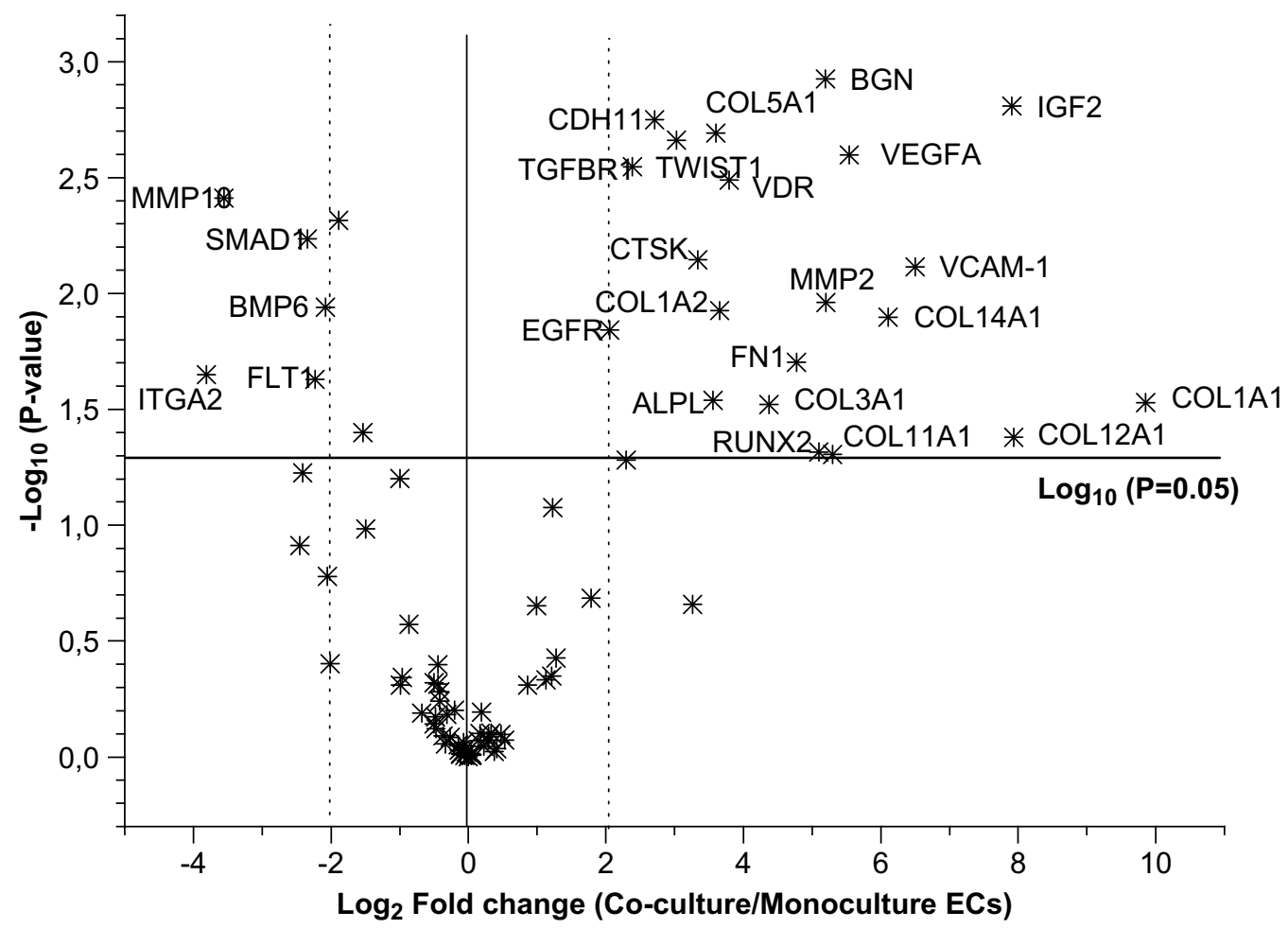

B

\begin{tabular}{|ll|}
\hline 0 & Co-culture versus Monoculture Osteoblasts \\
\hline
\end{tabular}

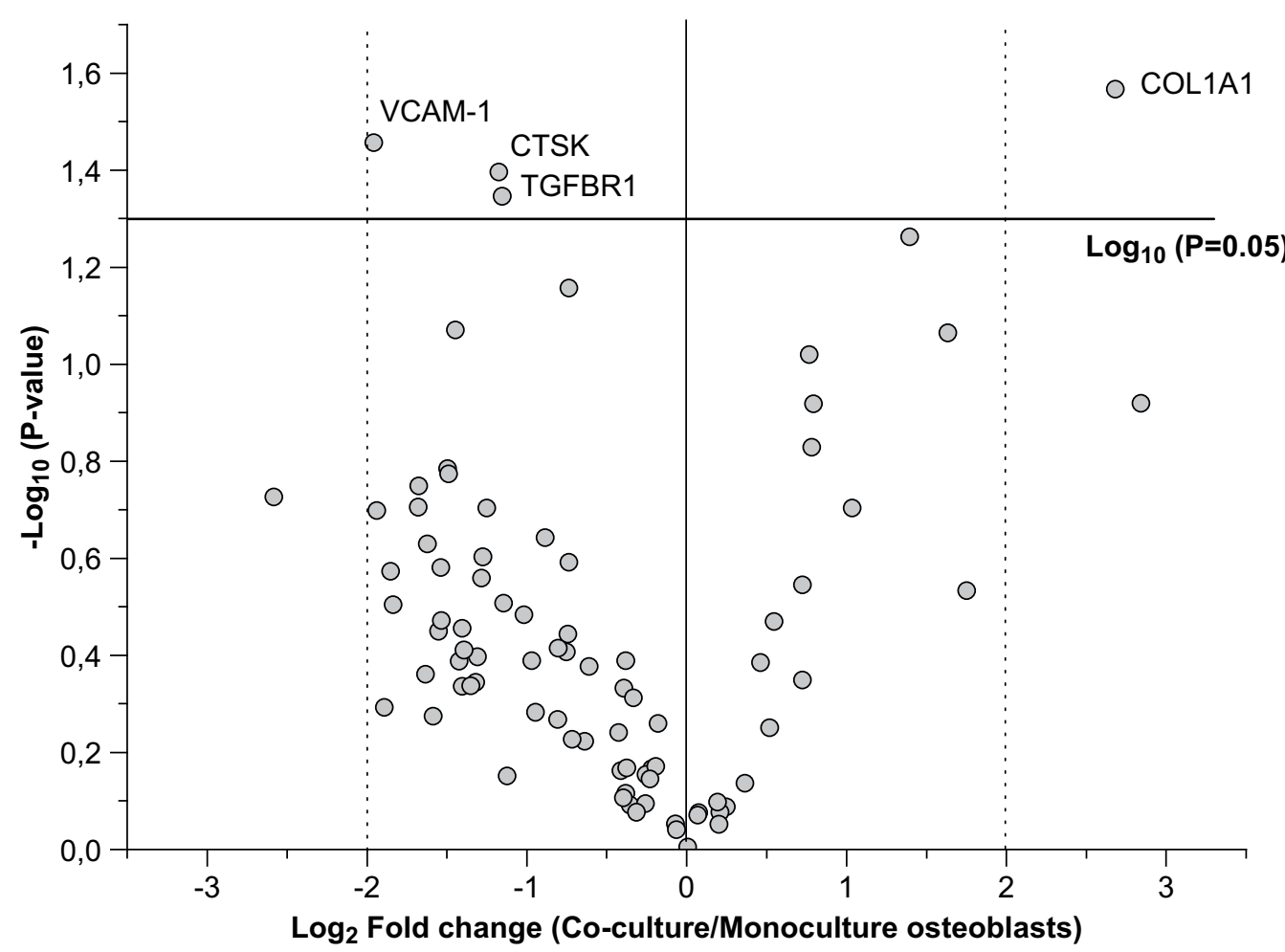

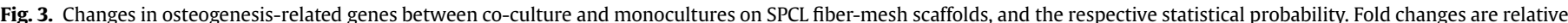

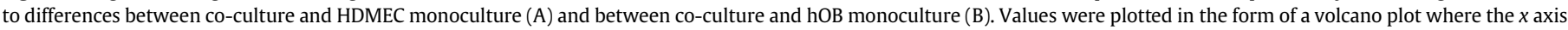

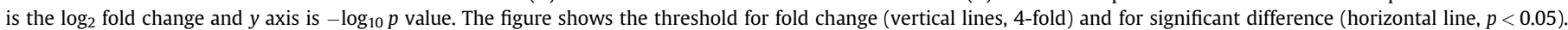

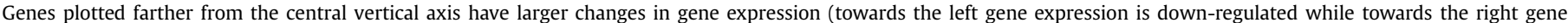
expression is up-regulated). The genes above the horizontal line are statistically significant. This data represent results from three different donors (ECs and osteoblasts). 
to basement membrane underlying ECs in blood vessels [26]. The formation of a structure with a lumen and the expression of the major constituent of the endothelial basement membrane assure the vascular nature of the established structures. However, microvessel stability requires crosstalk between EC and mural cells, such as pericytes [27]. Detailed studies in long-term culture are necessary to determine to what extent osteoblasts via a process of phenotypic change could adopt a pericyte and thus vessel stabilizing function. This is currently part of our further research activity.

The genetic profile of HDMEC:hOB co-culture compared to monocultures was also examined. Genetic profiling of co-cultures has also been explored by other authors [28,29]. Nevertheless, besides analyzing a limited number of the genes, the co-culture systems were different in many aspects (for example, ECs derived from macro-/microvasculature, 2D/3D cultures) and it is thus very difficult to compare such systems in a meaningful way, as multiple parameters vary. Thus, in scope of this work it was used the human osteogenesis PCR array to monitor the mRNA level of 84 osteogenesis-related genes in HDMEC:hOB co-culture, HDMEC and hOB monoculture, all cultured on SPCL fiber-mesh scaffolds. The assessment was performed after 21 days of culture, the time point when the first microcapillary-like structures were observed. Fig. 3 summarizes in the form of a Volcano plot the expression of each individual gene in co-culture vs. HDMEC monoculture (Fig. 3A) and co-culture vs. hOB monoculture (Fig. 3B), against the respective statistical significance ( $p$ value). Gene up-regulation was considered for a fold increase above 4 and considered statistically significant for $p<0.05$. In the case of co-culture vs. HDMEC monoculture, 5 down-regulated and 21 up-regulated genes were identified (Fig. 3A). Table 1 summarizes these genes grouped according to the biological processes they are involved in. The

Table 1

Genes regulated in co-culture relative to HDMEC monoculture. The genes are grouped according to the final biological function of the protein that they code for.

\begin{tabular}{|c|c|c|}
\hline Biological function & Symbol & Gene full name \\
\hline \multicolumn{3}{|l|}{ Down-regulated genes } \\
\hline \multirow[t]{2}{*}{$\begin{array}{l}\text { Growth factors and } \\
\text { receptors }\end{array}$} & BMP6 & $\begin{array}{l}\text { Bone morphogenetic } \\
\text { protein } 6\end{array}$ \\
\hline & Flt1 & $\begin{array}{l}\text { Vascular endothelial } \\
\text { growth factor receptor } 1\end{array}$ \\
\hline \multirow{2}{*}{$\begin{array}{l}\text { ECM-related and cell } \\
\text { adhesion molecules }\end{array}$} & MMP10 & Matrix metalloproteinase 10 \\
\hline & ITGA2 & Integrin alpha 2 \\
\hline Transcription factor & SMAD1 & SMAD family member 1 \\
\hline \multicolumn{3}{|l|}{ Up-regulated genes } \\
\hline \multirow[t]{5}{*}{$\begin{array}{l}\text { Growth factors and } \\
\text { receptors }\end{array}$} & VEGF & $\begin{array}{l}\text { Vascular endothelial } \\
\text { growth factor }\end{array}$ \\
\hline & IGF2 & Insulin-like growth factor 2 \\
\hline & VDR & Vitamin D receptor \\
\hline & EGFR & $\begin{array}{l}\text { Epidermal growth } \\
\text { factor receptor }\end{array}$ \\
\hline & TGF $\beta R 1$ & $\begin{array}{l}\text { Transforming growth } \\
\text { factor } \beta \text { receptor } 1\end{array}$ \\
\hline \multirow{12}{*}{$\begin{array}{l}\text { ECM-related and cell } \\
\text { adhesion molecules }\end{array}$} & Col IA1 & Collagen type I, alpha 1 \\
\hline & Col IA2 & Collagen type I, alpha 2 \\
\hline & Col IIIA1 & Collagen type III, alpha 1 \\
\hline & Col XIA1 & Collagen type XI, alpha 1 \\
\hline & Col XIIA1 & Collagen type XII, alpha 1 \\
\hline & Col5A1 & Collagen $\mathrm{V}$, alpha 1 \\
\hline & Col XIVA1 & Collagen type XIV, alpha 1 \\
\hline & CTSK & Cathepsin $\mathrm{K}$ \\
\hline & MMP2 & Matrix metalloproteinase 2 \\
\hline & FN1 & Fibronectin 1 \\
\hline & BGN & Biglycan \\
\hline & ALPL & Alkaline phosphatase \\
\hline \multirow{2}{*}{$\begin{array}{l}\text { Cell-cell adhesion } \\
\text { molecules }\end{array}$} & CDH11 & Cadherin 11 \\
\hline & VCAM-1 & Vascular cell adhesion molecule 1 \\
\hline \multirow[t]{2}{*}{ Transcription factors } & RUNX2 & Runt-related transcription factor 2 \\
\hline & TWIST1 & Twist transcription factor \\
\hline
\end{tabular}

down-regulated genes are related with extracellular matrix (ECM) and cell adhesion (MMP10, ITGA2), as well as with cell growth/ differentiation (SMAD1, BMP6, FLT1). In regard to up-regulated genes, there was a higher prevalence of genes that encode molecules related with ECM dynamics such as: structure (ex. Col IA1), cell adhesion (FN1), degradation (ex. CTSK) and mineralization (ALPL). Furthermore, many other genes were related with skeletal development such as growth factors (e.g. IGF2, TGFBR1) and transcription factors (RUNX2, TWIST1). Genes such as Col IA1 and IGF2, whose expression in co-culture increased 924- and 240-fold (respectively), had a very high threshold cycle (CT) value in the amplification plot of HDMEC monoculture, near to real-time PCR detection limit. Thus, this indicates these genes are almost not expressed in HDMEC. The observation of a very high up-regulation of insulin growth factor 2 (IGF2) in the co-cultures is also worthy of deeper investigation, as this growth factor is highly relevant for both osteoblast differentiated function and the process of angiogenesis [30,31]. Despite the up-regulation of the mRNA that encodes IGF2, the quantification of IGF2 at the protein level by ELISA in the supernatant of co-culture and monocultures gave no detectable levels. Whether the IGF2 mRNA is not being translated at all or whether the translated protein is unstable are questions that remain to be elucidated. Nevertheless, this dual function in both osteoblast proliferation and differentiation as well as angiogenesis makes IGF2 a potentially useful growth factor for bone tissue engineering and regeneration and thus this subject will be further studied. In the case of co-culture vs. hOB monoculture only Col I from the 84 genes under analysis had a significant up-regulation of 6.4-fold (Fig. 3B). The remaining genes showed no significant difference between hOBs cultured alone or co-cultured with HDMEC. To validate this result the expression of Col I was assessed by real-time RT-PCR. For 4 different donors, Col I was significantly $6.8 \pm 2.6$-fold up-regulated for hOBs co-cultured with HDMEC on SPCL fiber-mesh scaffolds, thus validating the microarray results. As previously described immunohistochemical studies revealed that spaces formed between the fibers of the micromesh scaffold were filled with a tissue-like dense network of Col I, and this was consistent with the results of the microarray analysis. Thus, the coculture of HDMECs with hOBs on SPCL fiber-mesh scaffold triggered Col I mRNA and protein synthesis. Col I is a marker for bone formation and accomplishes different functions from mediating cell adhesion to contributing to the mature osteoblast phenotype as well as providing a template for mineralization [32-34]. ECMassociated genes such as Col I are generally expressed during the proliferative period, whereas in heavily mineralized mature cultures (after 35 days) Col mRNA level is present at low levels [35]. Hence, since after 21 days of co-culture high levels of mRNA Col I are still observed, most probably hOBs are still in the proliferative phase. Moreover, mRNA coding for markers characteristic of other phases of bone cell differentiation such as osteonectin in ECM maturation and osteocalcin in mineralization [36] are not being produced in our co-culture system by hOBs at significant levels, otherwise their expression would be up-regulated on co-cultivation with ECs.

Col I is also known to drive EC migration by chemotaxis and haptotaxis [37]. The deposition of a very extensive network of Col I by hOBs in the scaffold provides an essential 3D support for ECs to migrate and organize into microcapillary-like structures. We believe that $\mathrm{Col} \mathrm{I}$ is a key factor for successful neovascularization in this co-culture system insofar as it provides ECs with the chemical and physical cues for migration and proliferation.

Another major issue in co-culture is the heterotypic intercellular crosstalk. Independent of the co-culture system used, it appears that communication between ECs and osteoblast cells occurs via diffusible factors as well as direct cell-cell contact 
$[8,9,28]$. For indirect cell communication, VEGF was the soluble factor examined in this study since it is a powerful pro-angiogenic factor with well established actions on ECs and with a proposed direct effect on osteoblast functions $[38,39]$. The concentration profile of VEGF produced in co-culture during the 35 days in vitro was characterized by three distinct phases: (i) from day 7 to 14 a steep increase in VEGF concentration; (ii) between day 14 and day 28 a plateau phase and (iii) from day 28 until day 35 a pronounced decrease of VEGF concentration (Fig. 4A). On the other hand, in hOB monoculture, the VEGF concentration curve exhibited a steady increase (but at lower magnitude as compared to co-culture) until day 28 followed by a decrease. The VEGF concentration in co-culture supernatant was 4 - to 2 times higher than in hOB monoculture $(p<0.05)$, while for HDMEC monoculture no VEGF was detected. These results indicate that hOBs in co-culture are stimulated to secrete higher amounts of VEGF. The results obtained for co-culture on SPCL fiber-mesh scaffolds were consistent with other reports, as osteoblasts were the predominant source of VEGF [40] and osteoblasts in co-culture released higher amounts of VEGF [8]. Furthermore, the release profile of VEGF with
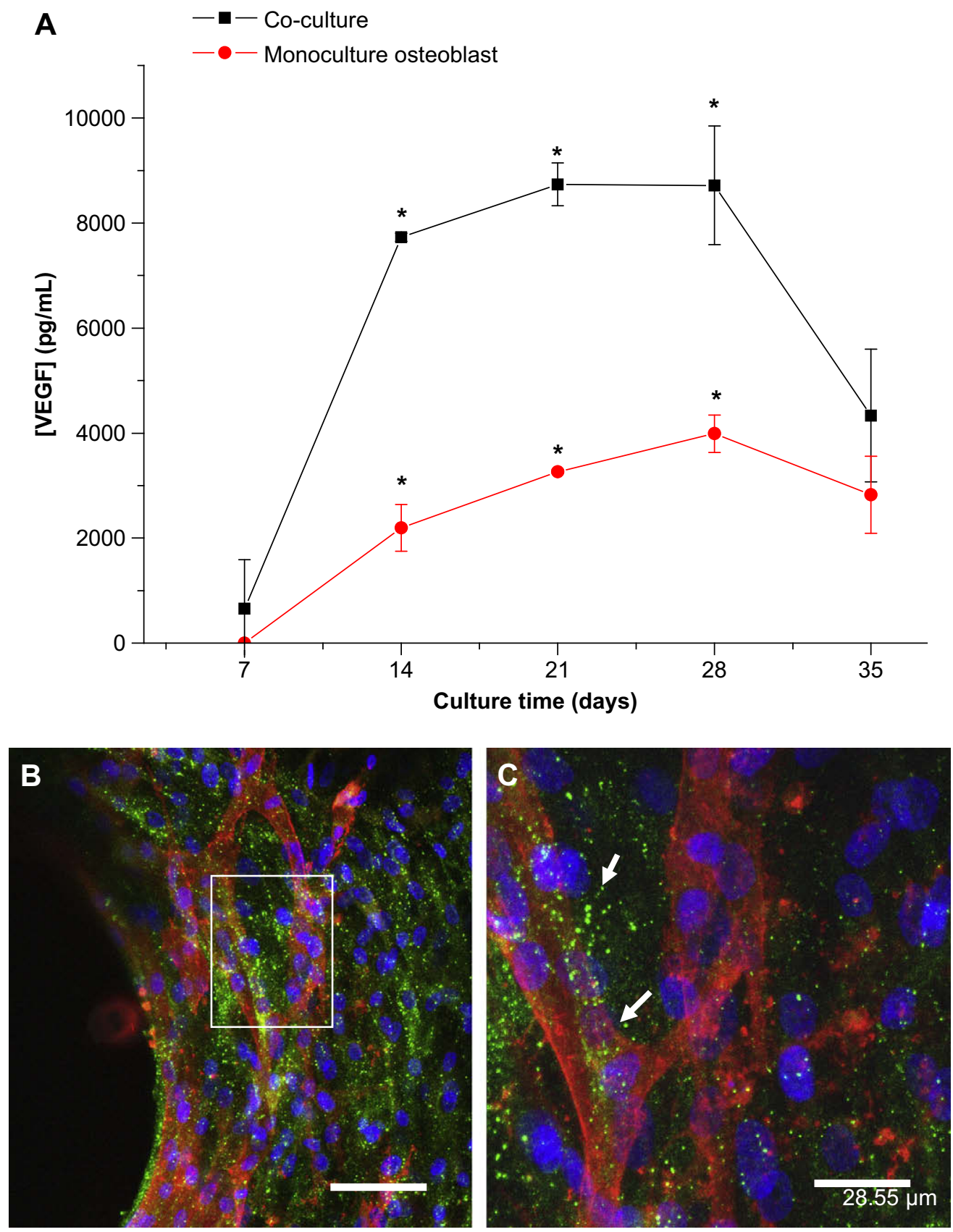

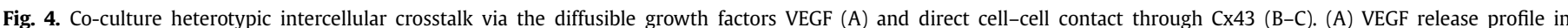

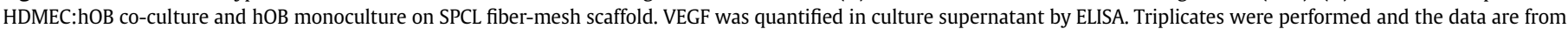

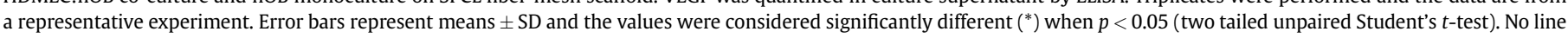

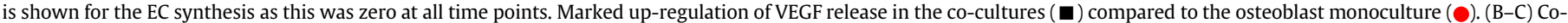

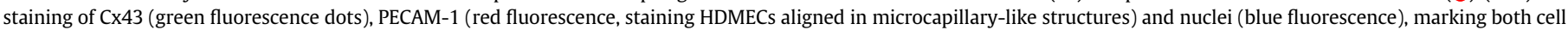

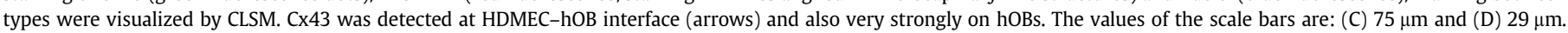


culture time revealed that the highest concentration peak of VEGF in co-culture coincided with the first observations of microcapillary-like structures. Recent studies [40] shed some light on the mechanisms responsible for controlling VEGF-dependent OB:EC crosstalk. These studies suggest a paracrine mechanism in which VEGF-stimulated ECs release prostaglandins that strongly promote the VEGF release in osteoblasts. For direct cell-cell contact the gap junction connexin $43(\mathrm{Cx} 43)$ was assessed in this co-culture system, due to its relevance for bone formation. In Fig. $4 \mathrm{~B}$ and $\mathrm{C}, \mathrm{Cx} 43$ is depicted as a punctuated bright green staining all over the co-culture section. Due to the co-staining of microcapillary-like structures (PECAM-1 staining, red fluorescence) and $\mathrm{Cx} 43$ it is possible to observe the expression of this gap junction protein at HDMEC-hOB interfaces (arrows) and in the areas where osteoblasts were depicted alone (only nuclear staining, blue fluorescence). Moreover, the osteoblasts also maintain a high expression of $\mathrm{Cx} 43$ in interaction with the scaffold material. Previous work [8] has proven that direct cell-cell contact is necessary for the formation of microcapillary-like structures by HDMECs and that these structures are not seen in cultures with conditioned medium or co-cultures with indirect contact, that is, separated by a porous synthetic membrane. Moreover, direct cellcell contact is a critical aspect of co-culture as the production of growth factors or gene expression is dependent on such contact $[9,29]$. Cx43 expression was also observed between hOBs. This is not surprising since it is well described in the literature that $\mathrm{Cx}_{43}$ is the major gap junction present in osteoblasts and it is known to modulate the expression of genes pivotal to bone matrix formation and calcification, such as bone sialoprotein and osteocalcin [41,42]. It is evident that further studies are necessary to investigate the time-related course of VEGF receptor expression in the EC, as well as much more detailed investigation of connexin expression in osteoblast-endothelial interactions need to be made.

\section{Conclusions}

The co-cultivation of HDMEC with hOBs on SPCL fiber-mesh scaffolds proved to be an effective strategy for the in vitro formation of microcapillary-like structures containing a lumen. Furthermore, the expression of Col IV and the evolution from cord-like configuration to a branched morphology confirmed the vascular nature and the complexity of the established microcapillary-like structures. Regarding the underlying mechanisms, the up-regulation of mRNA Col I in co-culture and the deposition of a dense ECM led us to postulate that, by providing chemical and physical cues for migrating ECs, Col I is a key molecule and modulator in this system. Moreover, the VEGF produced by co-cultured hOBs and the expression of the gap junction $\mathrm{Cx} 43$ between the two cell types indicated that heterotypic communication, a crucial aspect for coculture orchestration, was assured. Therefore, this strategy is defined as self-sustainable insofar as on SPCL fiber-mesh scaffolds HDMECs and hOBs recreate the physical and chemical microenvironment favorable for the formation of vascular-like structures, thus obviating the need for an exogenous supply of pro-angiogenic stimuli.

\section{Acknowledgements}

M.I. Santos would like to acknowledge the Portuguese Foundation for Science and Technology (FCT) for her PhD scholarship (SFRH/BD/13428/2003). This work was partially supported by FCT through funds from POCTI and/or FEDER programs and by the European Union funded STREP Project HIPPOCRATES (NMP3-CT2003-505758). This work was carried out under the scope of the European NoE EXPERTISSUES (NMP3-CT-2004-500283).
The authors would also like to acknowledge the excellent technical assistance of A. Sartoris. We greatly appreciate the help provided by Luciano Boesel with the statistical analysis.

\section{References}

[1] Klenke FM, Liu Y, Yuan H, Hunziker EB, Siebenrock KA, Hofstetter W. Impact of pore size on the vascularization and osseointegration of ceramic bone substitutes in vivo. J Biomed Mater Res A 2008;85(3):777-86.

[2] Koike N, Fukumura D, Gralla O, Au P, Schechner JS, Jain RK. Tissue engineering: creation of long-lasting blood vessels. Nature 2004;428(6979):138-9.

[3] Beilmann M, Birk G, Lenter MC. Human primary co-culture angiogenesis assay reveals additive stimulation and different angiogenic properties of VEGF and HGF. Cytokine 2004;26(4):178-85.

[4] Villars F, Bordenave L, Bareille R, Amedee J. Effect of human endothelial cells on human bone marrow stromal cell phenotype: role of VEGF? J Cell Biochem 2000;79(4):672-85.

[5] Brandi ML, Collin-Osdoby P. Vascular biology and the skeleton. J Bone Miner Res 2006;21(2):183-92.

[6] Deckers MML, van Bezooijen RL, van der Horst G, Hoogendam J, van der Bent C, Papapoulos SE, et al. Bone morphogenetic proteins stimulate angiogenesis through osteoblast-derived vascular endothelial growth factor A. Endocrinology 2002;143(4):1545-53.

[7] Villars F, Guillotin B, Amedee T, Dutoya S, Bordenave L, Bareille R, et al. Effect of HUVEC on human osteoprogenitor cell differentiation needs heterotypic gap junction communication. Am J Physiol Cell Physiol 2002;282(4):C775-85.

[8] Unger RE, Sartoris A, Peters K, Motta A, Migliaresi C, Kunkel M, et al. Tissuelike self-assembly in cocultures of endothelial cells and osteoblasts and the formation of microcapillary-like structures on three-dimensional porous biomaterials. Biomaterials 2007;28(27):3965-76.

[9] Wenger A, Stahl A, Weber H, Finkenzeller G, Augustin HG, Stark GB, et al. Modulation of in vitro angiogenesis in a three-dimensional spheroidal coculture model for bone tissue engineering. Tissue Eng 2004;10(9-10): 1536-47.

[10] Choong CS, Hutmacher DW, Triffitt JT. Co-culture of bone marrow fibroblasts and endothelial cells on modified polycaprolactone substrates for enhanced potentials in bone tissue engineering. Tissue Eng 2006;12(9):2521-31.

[11] Rouwkema J, De Boer J, Van Blitterswijk CA. Endothelial cells assemble into a 3-dimensional prevascular network in a bone tissue engineering construct. Tissue Eng 2006;12(9):2685-93.

[12] Fuchs S, Hofmann A, Kirkpatrick CJ. Microvessel-like structures from outgrowth endothelial cells from human peripheral blood in 2-dimensional and 3-dimensional co-cultures with osteoblastic lineage cells. Tissue Eng 2007;13(10):2577-88.

[13] Santos MI, Fuchs S, Gomes ME, Unger RE, Reis RL, Kirkpatrick CJ. Response of micro- and macrovascular endothelial cells to starch-based fiber meshes for bone tissue engineering. Biomaterials 2007;28(2):240-8.

[14] Santos MI, Tuzlakoglu K, Fuchs S, Gomes ME, Peters K, Unger RE, et al. Endothelial cell colonization and angiogenic potential of combined nano- and micro-fibrous scaffolds for bone tissue engineering. Biomaterials 2008;29(32):4306-13.

[15] Pavlov MP, Mano JF, Neves NM, Reis RL. Fibers and 3D mesh scaffolds from biodegradable starch-based blends: production and characterization. Macromol Biosci 2004;4(8):776-84.

[16] Gomes ME, Sikavitsas VI, Behravesh E, Reis RL, Mikos AG. Effect of flow perfusion on the osteogenic differentiation of bone marrow stromal cells cultured on starch-based three-dimensional scaffolds. J Biomed Mater Res 2003;67A(1):87-95.

[17] Gomes ME, Holtorf HL, Reis RL, Mikos AG. Influence of the porosity of starchbased fiber mesh scaffolds on the proliferation and osteogenic differentiation of bone marrow stromal cells cultured in a flow perfusion bioreactor. Tissue Eng 2006;12(4):801-9.

[18] Gomes ME, Bossano CM, Johnston CM, Reis RL, Mikos AG. In vitro localization of bone growth factors in constructs of biodegradable scaffolds seeded with marrow stromal cells and cultured in a flow perfusion bioreactor. Tissue Eng 2006;12(1):177-88.

[19] Tuzlakoglu K, Bolgen N, Salgado AJ, Gomes ME, Piskin E, Reis RL. Nano- and micro-fiber combined scaffolds: a new architecture for bone tissue engineering. J Mater Sci Mater Med 2005;16(12):1099-104.

[20] Gomes ME, Azevedo HS, Moreira AR, Ella V, Kellomaki M, Reis RL. Starchpoly(epsilon-caprolactone) and starch-poly(lactic acid) fibre-mesh scaffolds for bone tissue engineering applications: structure, mechanical properties and degradation behaviour. J Tissue Eng Regen Med 2008;2(5):243-52.

[21] Peters K, Schmidt H, Unger RE, Otto M, Kamp G, Kirkpatrick CJ. Softwaresupported image quantification of angiogenesis in an in vitro culture system: application to studies of biocompatibility. Biomaterials 2002;23(16): 3413-9.

[22] Annaz B, Hing KA, Kayser M, Buckland T, Di Silvio L. An ultrastructural study of cellular response to variation in porosity in phase-pure hydroxyapatite J Microsc 2004;216(Pt 2):97-109.

[23] Simon AM, McWhorter AR. Vascular abnormalities in mice lacking the endothelial gap junction proteins connexin37 and connexin40. Dev Biol 2002; 251(2):206-20. 
[24] Gerritsen ME, Soriano R, Yang SY, Zlot C, Ingle G, Toy K, et al. Branching out: a molecular fingerprint of endothelial differentiation into tube-like structures generated by affymetrix oligonucleotide arrays. Microcirculation 2003; 10(1):63-81.

[25] Bayless KJ, Salazar R, Davis GE. RGD-dependent vacuolation and lumen formation observed during endothelial cell morphogenesis in three-dimensional fibrin matrices involves the alpha(v)beta(3) and alpha(5)beta(1) integrins. Am J Pathol 2000;156(5):1673-83.

[26] Kuhn K. Basement membrane (type IV) collagen. Matrix Biol 1995;14(6): 439-45.

[27] Gaengel K, Genove G, Armulik A, Betsholtz C. Endothelial-mural cell signaling in vascular development and angiogenesis. Arterioscler Thromb Vasc Bio 2009;29(5):630-8.

[28] Finkenzeller G, Arabatzis G, Geyer M, Wenger A, Bannasch H, Stark GB. Gene expression profiling reveals platelet-derived growth factor receptor alpha as a target of cell contact-dependent gene regulation in an endothelial cellosteoblast co-culture model. Tissue Eng 2006;12(10):2889-903.

[29] Stahl A, Wenger A, Weber H, Stark GB, Augustin HG, Finkenzeller G. Bidirectional cell contact-dependent regulation of gene expression between endothelial cells and osteoblasts in a three-dimensional spheroidal coculture model. Biochem Biophys Res Commun 2004;322(2):684-92.

[30] Minuto F, Palermo C, Arvigo M, Barreca AM. The IGF system and bone J Endocrinol Invest 2005;28(8 Suppl.):8-10.

[31] Chao W, D'Amore PA. IGF2: epigenetic regulation and role in development and disease. Cytokine Growth Factor Rev 2008;19(2):111-20.

[32] Watts NB. Clinical utility of biochemical markers of bone remodeling. Clin Chem 1999;45(8 Pt 2):1359-68.

[33] Pham QP, Kasper FK, Scott Baggett L, Raphael RM, Jansen JA, Mikos AG. The influence of an in vitro generated bone-like extracellular matrix on osteoblastic gene expression of marrow stromal cells. Biomaterials 2008;29(18):2729-39.
[34] Lynch MP, Stein JL, Stein GS, Lian JB. The influence of type I collagen on the development and maintenance of the osteoblast phenotype in primary and passaged rat calvarial osteoblasts: modification of expression of genes supporting cell growth, adhesion, and extracellular matrix mineralization. Exp Cell Res 1995;216(1):35-45.

[35] Lian JB, Stein GS. Concepts of osteoblast growth and differentiation - basis for modulation of bone cell-development and tissue formation. Crit Rev Oral Biol Med 1992;3(3):269-305.

[36] Reuther T, Rohmann D, Scheer M, Kübler AC. Osteoblast viability and differentiation with $\mathrm{Me}_{2} \mathrm{SO}$ as cryoprotectant compared to osteoblasts from fresh human iliac cancellous bone. Cryobiology 2005;51(3):311-21.

[37] Davis GE, Senger DR. Endothelial extracellular matrix: biosynthesis, remodeling, and functions during vascular morphogenesis and neovessel stabilization. Circ Res 2005;97(11):1093-107.

[38] Li G, Cui YX, Mcllmurray L, Allen WE, Wang HL. RhBMP-2, rhVEGF(165), rhPTN and thrombin-related peptide, TP508 induce chemotaxis of human osteoblasts and microvascular-endothelial cells. J Orthop Res 2005;23(3): $680-5$.

[39] Roy H, Bhardwaj S, Yla-Herttuala S. Biology of vascular endothelial growth factors. FEBS Lett 2006;580(12):2879-87.

[40] Clarkin CE, Emery RJ, Pitsillides AA, Wheeler-Jones CP. Evaluation of VEGFmediated signaling in primary human cells reveals a paracrine action for VEGF in osteoblast-mediated crosstalk to endothelial cells. J Cell Physiol 2008; 214(2):537-44

[41] Lecanda F, Towler DA, Ziambaras K, Cheng SL, Koval M, Steinberg TH, et al. Gap junctional communication modulates gene expression in osteoblastic cells. Mol Biol Cell 1998;9(8):2249-58.

[42] Lecanda F, Warlow PM, Sheikh S, Furlan F, Steinberg TH, Civitelli R. Connexin43 deficiency causes delayed ossification, craniofacial abnormalities, and osteoblast dysfunction. J Cell Biol 2000;151(4):931-44. 